\title{
Atuação do enfermeiro no gerenciamento dos eventos adversos realacioandos aos cateter venoso central: revisão integrativa
}

Nurse's role in managing adverse events related to central venous catheters: integrative review Papel de la enfermera en el manejo de eventos adversos relacionados con catéteres venosos centrales: revisión integradora

Adriana Feitoza Pereira

ORCID: https://orcid.org/0000-0002-1141-7210 Centro Universtirário Estácio de Sergipe, Brasil E-mail: adryfeitoza_89@outlook.com

Ana Fatima Souza Melo de Andrade ORCID: https://orcid.org/0000-0002-7024-6175 Centro Universitário Estácio de Sergipe, Brasil E-mail: anafatimamelo@hotmail.com

Weber de Santana Teles ORCID: https://orcid.org/0000-0003-1770-8278 Instituto de Hemoterapia de Sergipe, Brasil

E-mail: arteecura@hormail.com

Max Cruz da Silva

ORCID: https://orcid.org/0000-0002-6944-5986

Faculdade Pio Décimo, Brasil

E-mail: maxlfi@hotmail.com

Ruth Cristini Torres

ORCID: https://orcid.org/0000-0002-8664-192X Instituto de Hematologia e Hemoterapia de Sergipe, Brasil

E-mail: ruthcristini@gmail.com

Ângela Maria Melo Sá Barros

ORCID: https://orcid.org/0000-0003-4087-3247

Universidade Federal do Rio de Janeiro, Brasil

E-mail: angelsamelo@hotmail.com

Marcel Vinícius Cunha Azevedo

ORCID: https://orcid.org/0000-0002-5312-3333

Centro Universitário Estácio Sergipe, Brasil E-mail: marcelvinicius49@gmail.com

Alejandra Debbo

ORCID: https://orcid.org/0000-0002-7743-5921 Universidade Federal de Sergipe, Brasil

E-mail: aledebbo@hotmail.com

André Luiz de Jesus Morais

ORCID: https://orcid.org/0000-0003-4889-8297

Centro Universitário Estácio de Sergipe, Brasil

E-mail: enfermeiro.andre@ hotmail.com

Maria Hozana Santos Silva

ORCID: http://orcid.org/0000-0001-5742-5366

Faculdade Ages de Medicina, Brasil

E-mail: hosana_p@hotmail.com

Taíssa Alice Soleade Calasans

ORCID: https://orcid.org/0000-0003-0460-4437

Universidade Tiradentes, Brasil

E-mail: taissa.asc@gmail.com

Paulo Celso Curvelo Santos Junior

ORCID: https://orcid.org/0000-0001-5834-6782

Universidade Tiradentes, Brasil

E-mail: paulo.curvelo.jr@gmail.com

\section{Resumo}

O Cateter Venoso Central é um dispositivo intravascular, cuja extremidade distal está situada próxima ou no interior do átrio direito, podendo ser inserida também em vasos de grande calibree tem como finalidade possibilitar a infusão venosa contínua ou intermitente, administração de hemocomponentes e hemoderivados, nutrição parenteral, entre outros. Este estudo tem como objetivo descrever a atuação do enfermeiro na gestão do cuidado e gerenciamento de eventos adversos associados ao cateter venoso central. Trata-se de uma revisão bibliográfica da literatura, cujos artigos 
foram filtrados nas bases de dados: Literatura Latino-Americana e do Caribe em Ciências da Saúde (LILACS), Scientific Electronic Library Online (SciELO), PubMed, Base de Dados de Enfermagem (BDENF) e Google Acadêmico. O estudo evidenciou a importância de gerenciar riscos inerentes à cateterização, desde sua inserção até remoção. A infecção da correntes sanguínea gastou destaque como um dos principais eventos. Destacou-se também a necessidade da utilização debundles, enfatizando a importância da cultura de segurança. Portanto, conclui-se que o enfermeiro tem um papel essencial na prevenção e controle de infecções, pois assiste diretamente os pacientes, manipulam e controlam dispositivos, conexões e medicações. A utilização de bundles são eficazes na redução das taxas de infecção.

Palavras-chave: Cateteres; Eventos adversos; Infecções relacionadas a cateter; Cuidados de enfermagem.

\begin{abstract}
The Central Venous Catheter is an intravascular device, the distal end of which is located close to or inside the right atrium, and can also be inserted into large-caliber vessels and aims to enable continuous or intermittent venous infusion, administration of blood components and blood products, nutrition parenteral, among others. This study aims to describe the role of nurses in the management of care and management of adverse events associated with the central venous catheter. It is a bibliographic review of the literature, whose articles were filtered in the Latin American and Caribbean Literature on Health Sciences (LILACS), Scientific Electronic Library Online (SciELO), PubMed, Nursing Database (BDENF) and Academic Google. The study highlighted the importance of managing risks inherent to catheterization, from insertion to removal. Bloodstream infection has become prominent as one of the main events. The need to use bundles was also highlighted, emphasizing the importance of a safety culture. Therefore, it is concluded that nurses have an essential role in the prevention and control of infections, as they directly assist patients, manipulate and control devices, connections and medications. The use of bundles are effective in reducing infection rates.
\end{abstract}

Keywords: Catheters; Adverse events; Catheter-related infections; Nursing care.

\title{
Resumen
}

El catéter venoso central es un dispositivo intravascular, cuyo extremo distal está ubicado cerca o dentro de la aurícula derecha, que también se puede insertar en vasos de gran calibre y está destinado a permitir la infusión venosa continua o intermitente, la administración de componentes sanguíneos y productos sanguíneos, nutrición parenteral, entre otros. Este estudio tiene como objetivo describir el papel de las enfermeras en el manejo de la atención y el manejo de los eventos adversos asociados con los catéteres venosos centrales. Se trata de una revisión de la literatura, cuyos artículos fueron filtrados en las bases de datos Literatura Latinoamericana y del Caribe en Ciencias de la Salud (LILACS), Scientific Electronic Library Online (SciELO), PubMed, Nursing Database (BDENF) y Academic Google. El estudio destacó la importancia de gestionar los riesgos inherentes al cateterismo, desde la inserción hasta la extracción. La infección del torrente sanguíneo se destacó como uno de los principales eventos. También se destacó la necesidad de utilizar paquetes, haciendo hincapié en la importancia de una cultura de seguridad. Por tanto, se concluye que las enfermeras tienen un papel fundamental en la prevención y control de infecciones, ya que asisten directamente a los pacientes, manipulan y controlan dispositivos, conexiones y medicamentos. El uso de paquetes es eficaz para reducir las tasas de infección.

Palabras clave: Catéteres; Eventos adversos; Infecciones relacionadas con el catéter; Cuidado de enfermera.

\section{Introdução}

O Cateter Venoso Central (CVC) consiste num dispositivo intravascular, cuja extremidade distal está situada próxima ou no interior do átrio direito, podendo ser inserida também em vasos de grande calibre. Os principais objetivos deste dispositivo são coleta de sangue, infusão venosa contínua ou intermitente; administração de hemocomponentes e hemoderivados; nutrição parenteral; administração de medicamentos que, em virtude da osmolaridade e outras especificidades, não admitem a utilização da via periférica; além de ser utilizado para mensuração da pressão venosa central (Centers For Disease Control And Prevention, 2016).

Segundo Danski et al. (2017), o uso de CVC não é isento de complicações eaos menos $15 \%$ dos pacientes desenvolvem uma delas, o que inclui eventos infecciosos e não infecciosos como: Infecções da Corrente Sanguínea Relacionada ao Cateter (ICSRC), pneumotórax, hemotórax, perda de cateter e hematoma. Outros eventos adversos podem surgir, como infiltração, embolia gasosa, arritmia cardíaca, localização inadequada da ponta do cateter ou lesão de estruturas vizinhas.

Segundo os dados do Centers for Disease Control and Prevention (CDC), as ICSRC são Eventos adveros (EA) que resultam em aumento do tempo de internação (em média 12 dias) edos custos hospitalares, com prejuízos de até 2,3 bilhões de dólares anuais. As taxasde mortalidade associadas a esse evento variam de 10 a 30\%, nos EUA e de 6,7 a 75\%, no Brasil 
(Marques, Aquino \& Júnior, 2019).

Para Oliveira \& Rodas (2017), as possíveis consequências dos eventos adversosrelacionados ao uso do CVC, tais como o agravo do prognóstico do paciente, o aumento do tempo de internação e do uso de antibióticos, destaca-se a importância da padronização das ações dos profissionais de saúde com o objetivo de reduzir as taxas desses eventos, dessa maneira, o conhecimento aprofundado em eventos adversos relacionados ao uso CVC, no ambiente hospitalar, é de suma importância para acompreensão dos fatores que contribuem e influenciam sua ocorrência, com consequente elaboração de medidas para contêlos.

A participação do enfermeiro na implantação de estratégias para a melhoria da qualidade e da segurança da assistência de enfermagem torna-se indispensável na tentativa de subsidiar os profissionais da área, ou seja, fornece suporte para que estes conheçam as causas e os efeitos à saúde do cliente, além de possibilitar treinamentos adequados à prevenção de novos eventos adversos e construção da cultura de segurança nos serviços de saúde, de forma ampliada (Ribeiro, et al., 2020).

O enfermeiro é ator responsável no gerenciamento do cateter. Porém, isso não deve limitar-se à valorização dos procedimentos apenas como técnica, e sim englobar os diversos conhecimentos técnico-científicos que fundamentam essa prática. Atrelado a isso, a implementação de protocolos, normas, rotinas, Procedimento Operacional Padrão (POP), funcionam como diretrizes e fornecem um respaldo técnico para o profissional instituir um procedimento prescrito, seguindo normas e recomendações internacionais a respeito do assunto (Silva, et al., 2018).

De acordo com Rynga et al. (2017) uma das estratégias para limitar o avanço das infecções relacionadas ao cateter ou até mesmo a ICS é a adoção de bundle (conjunto de protocolos multidisciplinares para a assistência de qualidade) que se revela um grande desafio para as instituições, desprincipalmente, porque envolve a Cultura de Segurança e de outras características da instituição que refletem, inclusive, em maiores gastos financeiros.

Os eventos adversos (EA) são incidentes e frequentemente resultam em dano, se caracterizam como situações de caráter deletério, as quais concorrem para as práticas de imperícia, imprudência e negligência, ou seja, tratam-se de ocorrências diretamente relacionadas aos cuidados de saúde prestados ao indivíduo, sobre o qual sua ocorrência pode ou não produzir danos, aumentando o tempo de internação. Estima-se que 1 em cada 10 pacientes no mundo é vítima de EA evitáveis durante o período que necessita receber assistência integral (Roque, Tonini \& Melo, 2016).

De acordo com Ramírez, et al., (2017), os EA estão geralmente associados ao erro humano individual, porém devem ser considerados como produtos de um sistema complexo que envolve uma complexidade de processos de trabalho, cujo atores apresentam características profissionais distintas; uma infraestrutura que esteja inserida num clima organizacional conturbado; a necessidade de uma gestão administrativa sensível à capacitação permanente dos recursos humanos, bem como, a Cultura de Segurança adotada pela instituição. Além disso, as situações que predispõem ao risco de EA incluem a delegação de cuidados sem supervisão adequada e sobrecarga de serviço.

No que se refere à finalidade do CVC, bem como, a natureza das soluções introduzidas por este cateter, é indispensável trazer à discussão a ocorrência de infecções relacionadas ao uso deste, sobre o qual derivam diversas complicações, entre elas o pneumotórax, pneumonia, trombose venosa profunda (TVP), infecção da corrente sanguínea relacionada ao cateter (ICSRC), embolia gasosa, entre outras, revelando-se um problema de grande magnitude. Assim, calcula-se que em torno de $90 \%$ das infecções de corrente sanguínea (ICS) são decorrentes do uso de CVC, sendo esta um dos quatro sítios com maior importância no controle de infecção e com elevado custo para os serviços de saúde (Weeks, et al., 2011).

Para Barbosa, et al., (2020), a punção venosa central não se trata de um procedimento isento de risco, isto é, existe uma relação direta entre o índice de complicações e a experiência do cirurgião e dos profissionais que irão manuseá-la. Desta forma, verifica-se a ocorrência de punção arterial acidental, pneumotórax, hemotórax, tamponamento cardíaco, hematoma local, infecções, embolia e hidrotórax como repercussões correlatas à punção. 
Em se tratando da ocorrência de EA, torna-se indispensável a discussão sobre a Segurança do Paciente e as Boas Práticas para efetivação desta. Sendo assim, em 2002, a Organização Mundial da Saúde (OMS) reconheceu como prioridade os problemas relacionados à assistência segura do paciente. Em função disso, este organismo internacional criou em 2004 a Aliança Mundial para Segurança do Paciente, que tem por objetivo definir metas acerca do tema e contribuir para elaboração de uma agenda mundial em pesquisas (World Health Organization, 2009).

As Metas Internacionais de Segurança do Paciente estão contidas na RDC n ${ }^{\circ}$ 63/11, que trata sobre os requisitos de boas práticas de funcionamento dos serviços de saúde. Esta resolução traz em seu artigo $8^{\circ}$ que esses estabelecimentos devem propor estratégias e ações voltadas à Segurança do Paciente, tais como: orientações para a higienização das mãos; ações de prevenção e controle de EA; programas de educação permanente visando a oferta de procedimentos cirúrgicos seguros; recomendações para administração segura e efetiva de fármacos e hemocomponentes; e aconselhamento para incentivar a participação do cliente na assistência prestada, sob à ótica do cuidado compartilhado (Brasil, 2011).

Em consonância com as metas internacionais elencadas pela Organização Mundial da Saúde (OMS), o Ministério da Saúde através da Anvisa baixou a Resolução - $\operatorname{RDC}^{\circ}$ 36, de 25 de julho de 2013 que dispõe sobre a instituição de ações para a segurança do paciente em serviços de saúde, sintetizando alguns conceitos inerentes às Boas Práticas para Segurança do Paciente, que têm como focos de atuação a prevenção e minoração de eventos desagradáveis ao usuário do serviço, entre eles os eventos adversos, os quais podem determinam debilidade temporária ou permanente de membro, sentido ou função ou até mesmo o óbito. Alguns conceitos serão apresentados a seguir:

Art. $3^{\circ}$ Para efeito desta Resolução são adotadas as seguintes definições:

I - boas práticas de funcionamento do serviço de saúde: componentes da garantia da qualidade que asseguram que os serviços são ofertados com padrões de qualidade adequados (Brasil, 2013, p. 1);

- dano: comprometimento da estrutura ou função do corpo e/ou qualquer efeito dele oriundo, incluindo doenças, lesão, sofrimento, morte, incapacidade ou disfunção, podendo, assim, ser físico, social ou psicológico (Brasil, 2013, p. 1); - evento adverso: incidente que resulta em dano à saúde (Brasil, 2013, p. 1);

VI - gestão de risco: aplicação sistêmica e contínua de políticas, procedimentos, condutas e recursos na identificação, análise, avaliação, comunicação e controle de riscos e eventos adversos que afetam a segurança, a saúde humana, a integridade profissional, o meio ambiente e a imagem institucional (Brasil, 2013, p. 2);

$\mathrm{X}$ - segurança do paciente: redução, a um mínimo aceitável, do risco de dano desnecessário associado à atenção à saúde (Brasil, 2013, p. 2).

A RDC n 36/2013 engloba em suas propostas as ações de notificação e monitoramento de EA atinentes aos produtos, bem como, às falhas nos processos de cuidado, o que irá possibilitar a ampliação e a articulação do conjunto de notificações de EA no país. Essa realidade traz à tona a necessidade de organização desse relevante processo de trabalho realizado no âmbito do Sistema Nacional de Vigilância Sanitária - (SNVS). bem como, suscita a importância de um diálogo construtivo e, sobretudo, resolutivo entre as unidades assistenciais que integram as Redes de Atenção à Saúde, a fim de reduzir a ocorrência dos EA, além de permitir a detecção de situações e características do serviço que dão margem à subnotificação dos casos (Brasil, 2014).

Vale ressaltar que todos os EA relatados neste estudo deve ser notificado. A notificação efetuada no Notivisa é integralizada por 10 etapas: 1) Tipo de incidente/evento; 2) Repercussões para o cliente; 3) Características do paciente; 4) Especificidades do incidente/evento adverso; 5) Fatores associados; 6) Desdobramentos organizacionais; 7) Identificação; 8) Circunstâncias atenuantes do dano; 9) Ações de melhoria e 10) Ações para minimizar o risco. Cada etapa possui uma gama de variáveis objetivas e estruturadas, com vistas à classificação e refinamento qualitativo do EA. Esses conceitos encontram-se descritos, de forma pormenorizada, na publicação da Anvisa denominada "Implantação do Núcleo de Segurança do Paciente em Serviços de Saúde” (Brasil, 2016).

Dessa forma, este estudo tem como objetivo descrever a atuação do enfermeirona gestão do cuidado e gerenciamento de EA associados ao CVC. 


\section{Metodologia}

O presente estudo trata-se de uma revisão bibliográfica da literatura, de caráter exploratório-descritivo com abordagem qualitativa. Para a elaboração da pesquisa, foram seguidas as seguintes etapas: identificação do tema, estabelecimento dos critérios de inclusão e exclusão dos artigos; definição de dados que seriam extraídos, e finalmente a apresentação da revisão. A revisão de literatura consiste no processo de busca, análise e descrição de um corpo do conhecimento visando responder uma pergunta específica, ou seja, a pergunta de pesquisa. Este tipo de estudo contempla todo material relevante que é escrito sobre um tema: livros, artigos de periódicos, artigos de jornais, registros históricos, relatórios governamentais, teses e dissertações e outros tipos (Marconi \& Lakatos, 1992).

Os critérios de inclusão contemplaram os estudos primários publicados naíntegra nas bases de dados supracitadas, entre 2016 e 2020, aqueles que responderama ao objetivo da pesquisa, artigos disponíveis em dados de domínio público, publicados em português, inglês e espanhol. Os critérios de exclusão foram: Publicações incompletas, estudos duplicados e que não foram disponibilizados gratuitamente. Foram utilizados os descritores (DeCS): Cateteres; Eventos adversos; Infecções relacionadas a cateter; Complicações, Cuidados de enfermagem e Segurança do paciente, onde foram cruzados com os operadores OR e AND.

Foi utilizada a seguinte estratégia de busca: (cateter OR infecção OR eventos adversos OR complicações AND cuidados de enfermagem OR segurança do paciente).

A presente investigação foi realizada através da busca de artigos científicos publicados em português, inglês e espanhol disponíveis nas bases de dados LILACS (Literatura Latino-Americana e do Caribe em Ciências da Saúde), SciELO (Scientific Eletronic Library Online), PubMed, BDENF (Base de Dados de Enfermagem) e Google Acadêmico.

Para análise da amostra dos artigos incluídos na pesquisa foi elaborado um quadro síntese, o qual avaliou informações importantes de cada artigo e de interesse das pesquisadoras. A elaboração deste instrumento permitiu agregar informações relevantes, a fim de responder a questão norteadora desta revisão, composto pelos seguintes itens: autores e ano, revista ou periódico, título, objetivo e desenho doestudo.

\section{Resultados e Discussão}

Foram rastreados nas bases de dados LILACS, SciELO, PubMed, BDENF e Google Acadêmico um total de 735 artigos. Destes, 429 estudos foram excluídos apósa aplicação dos critérios de inclusão e exclusão. Em seguida, 157 artigos foram excluídos após a leitura dos títulos; 78 após a leitura dos resumos, restando 71 estudos elegíveis. Destes, 64 foram excluídos após a leitura na íntegra, totalizando 7 artigos para elaboração do quadro síntese. Como demonstra a Figura 1. 
Figura 1: Fluxograma na modalidade Prisma da seleção de publicações.

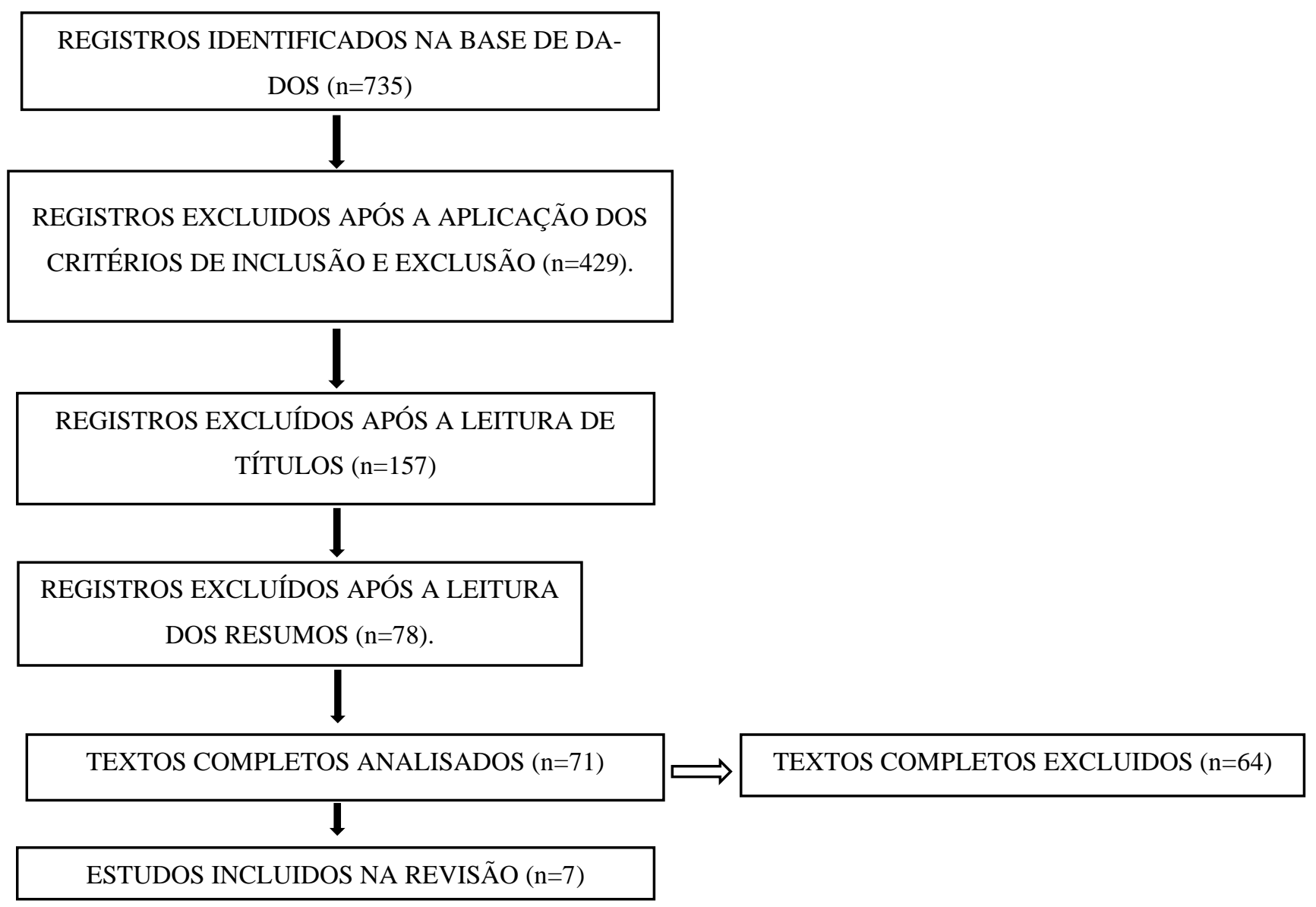

Fonte: Autores.

A partir disso, foi possível analisar os artigos integrando as ideias dos autores, permitindo a realização de uma análise integrativa e coerente relacionada com o objetivo do presente estudo, como demonstrado no Quadro 1. 
Quadro 1: Apresentação dos estudos selecionados.

\begin{tabular}{|c|c|c|c|c|}
\hline $\begin{array}{c}\text { AUTOR } \\
\text { ANO }\end{array}$ & REVISTA & TÍTULO & OBJETIVO & $\begin{array}{l}\text { DESENHO DO } \\
\text { ESTUDO }\end{array}$ \\
\hline Ortega & Acta Paulista de & $\begin{array}{c}\text { Análise de eventos adversos } \\
\text { En pacientes internados em } \\
\text { unidade de terapia } \\
\text { intensiva }\end{array}$ & $\begin{array}{c}\text { Avaliar a incidência de } \\
\text { los ao dimensionamento da } \\
\text { perfil de gravidade do } \\
\text { paciente }\end{array}$ & $\begin{array}{c}\text { Estudo } \\
\text { prospectivo }\end{array}$ \\
\hline Sousa & $\begin{array}{l}\text { Revista de } \\
\text { em Saúde }\end{array}$ & $\begin{array}{l}\text { Avaliação dos cuidados de } \\
\text { enfermagem com o cateter } \\
\text { Venoso central em uma } \\
\text { unidade de terapia } \\
\text { intensiva adulto e pediatrica }\end{array}$ & $\begin{array}{c}\text { Avaliar os cuidados de } \\
\text { cateter venoso central (CVC) } \\
\text { em uma Unidade de Terapia }\end{array}$ & Estudo do tipo \\
\hline $\begin{array}{c}\text { Costa } \\
\text { et al. }(2020)\end{array}$ & $\begin{array}{l}\text { Revista Escola de } \\
\text { EnfermagemUSP }\end{array}$ & $\begin{array}{l}\text { Bundle de CateterVenoso } \\
\text { Central: conhecimento e } \\
\text { comportamento de } \\
\text { profissionais em Unidades } \\
\text { de Terapia Intensiva adulto }\end{array}$ & $\begin{array}{l}\text { Avaliar o conhecimento e o } \\
\text { comportamento dos } \\
\text { profissionais de Unidade de } \\
\text { Terapia Intensiva no que se } \\
\text { refere às ações recomendadas } \\
\text { em bundles de prevenção de } \\
\text { IPCS associada ao uso do CVC }\end{array}$ & $\begin{array}{l}\text { Estudo } \\
\text { transversal, } \\
\text { descritivo }\end{array}$ \\
\hline $\begin{array}{c}\text { Silva } \\
\text { et al. }(2020)\end{array}$ & $\begin{array}{l}\text { Global Academic } \\
\text { Nursing Journal }\end{array}$ & $\begin{array}{c}\text { Indicador de flebitee } \\
\text { cuidados de enfermagem em } \\
\text { crianças e adolescentes com } \\
\text { cateter central de inserção } \\
\text { periférica }\end{array}$ & $\begin{array}{l}\text { Analisar a prevalência de flebite } \\
\text { em crianças e adolescentes que } \\
\text { fizeram uso de cateter venoso } \\
\text { periférico e cateter central de } \\
\text { inserção periférica }\end{array}$ & $\begin{array}{l}\text { Estudo } \\
\text { exploratório- } \\
\text { descritivo, } \\
\text { retrospectivo e } \\
\text { documental }\end{array}$ \\
\hline $\begin{array}{l}\text { Costa et al. } \\
\quad(2016)\end{array}$ & $\begin{array}{l}\text { Acta Paulista de } \\
\text { Enfermagem }\end{array}$ & $\begin{array}{c}\text { Fatores de risco para infecção } \\
\text { de corrente sanguínea } \\
\text { associada ao cateter central } \\
\text { de inserção periféricaem } \\
\text { neonatos }\end{array}$ & $\begin{array}{l}\text { Identificar os fatores de riscopara } \\
\text { infecção de corrente sanguínea } \\
\text { associada ao cateter central de } \\
\text { inserção periférica em neonatos }\end{array}$ & $\begin{array}{l}\text { Estudo de } \\
\text { coorte } \\
\text { prospectivo }\end{array}$ \\
\hline $\begin{array}{l}\text { Llapa- } \\
\text { Rodríguez et } \\
\text { al. }(2020)\end{array}$ & $\begin{array}{c}\text { Texto \& } \\
\text { Contexto- } \\
\text { Enfermagem }\end{array}$ & $\begin{array}{l}\text { Segurança na trocade equipos } \\
\text { e curativos para cateter } \\
\text { vascular central: um estudo } \\
\text { observacional }\end{array}$ & $\begin{array}{l}\text { Avaliar a conformidade da prática } \\
\text { assistencial da equipe de } \\
\text { enfermagem no manuseio do } \\
\text { cateter vascularcentral na troca de } \\
\text { curativos e de equipos em } \\
\text { unidade deterapia intensiva }\end{array}$ & $\begin{array}{l}\text { Pesquisa } \\
\text { descritiva, } \\
\text { prospectiva, } \\
\text { observacional }\end{array}$ \\
\hline $\begin{array}{c}\text { Rangel } \\
\text { et al. (2019) }\end{array}$ & $\begin{array}{l}\text { Revista } \\
\text { Pesquisa } \\
\text { Cuidado } \\
\text { Fundamental } \\
\text { (Online) }\end{array}$ & $\begin{array}{l}\text { Práticas de inserção, } \\
\text { manutenção e remoção do } \\
\text { CateterCentral de Inserção } \\
\text { Periférica emneonatos }\end{array}$ & $\begin{array}{c}\text { Avaliar as práticas de } \\
\text { enfermagem na inserção, } \\
\text { manutenção e remoção do Cateter } \\
\text { Central de InserçãoPeriférica em } \\
\text { neonatos }\end{array}$ & $\begin{array}{l}\text { Estudo } \\
\text { correlacional } \\
\text { retrospectivo }\end{array}$ \\
\hline
\end{tabular}

Fonte: Autores.

A perda de cateter central de inserção periférica (PICC) e a perda de cateter venoso central (CVC) foram os EA evidenciados com maior frequência. Atrelado a isso, estudo prévio evidenciou uma maior incidência de EA associados a dispositivos intravasculares (56\%), seguido de erros com medicação em $43 \%$ dos casos. Isso denota a importância de o enfermeiro gerenciar os riscos inerentes ao CVC, que podem ser: formação de trombos, embolias e infecções de corrente sanguínea, sendo assim, a equipe de enfermagem deve estar atenta à manipulação e manutenção dos CVC, desde sua inserção até sua remoção (Ortega, 2017).

O estudo de Sousa, et al., (2018) demonstrou que todos os curativos de CVCforam realizados pelos enfermeiros, sendo que a cobertura com filme poliuretano estéril foi empregada em $60 \%$ dos CVC. Entretanto, nenhum possuía a data de punção do 33,3\% não possuíam a data de realização do curativo anterior. Sendo assim, ressalta- se que a técnica asséptica utilizada pelos enfermeiros foi inadequada em 100\% dos curativos. Neste sentido, preconiza-se que o tempo de aplicação da clorexidina alcoólica seja de 30 segundos e seja efetuada através de movimentos de vai e vem, com gaze trocada de lado a cada 
pincelada, a fim de promover a secagem espontânea do antisséptico.

Diante do exposto, convém destacar que o enfermeiro detém um papel essencial na prevenção da ICSRC, visto que a manipulação do cateter, principalmentea troca de curativo, é de responsabilidade deste profissional, devendo responsabilizarse, inclusive, pela identificação e notificação dos casos de infecção associada aos dispositivos intravasculares, visando atuar na profilaxia e no controle das infecções relacionadas à assistência à saúde (IRAS).

Verificou- se maior discordância entre os médicos quanto ao uso de barreiras máximas de proteção ser um item do bundle, quer seja para o profissional que insere o cateter ou para a aquele que auxilia a inserção. Em relação à degermação da pele, a menor resposta de concordância foi observada entre os técnicos enfermagem. Já os enfermeiros mostraram-se mais assertivos quando comparados aos demais grupos. Todavia, o estudo chama atenção para o fato de que "datar conectores" predominou no grupo de médicos, haja vista que se trata de uma ação frequentemente atribuída à enfermagem (Costa, 2020).

Observou-se que o grupo dos enfermeiros apresentou maior percentual da frequência de que "algumas vezes" realizam a limpeza do hub ou conectores comálcool a 70\% em comparação aos demais grupos. Isso demonstra que o enfermeiro deve estar capacitado para avaliar a integridade dos acessos, para identificar os eventos adversos associados ao cateter, bem como, ter domínio sobre as novas coberturas e dispositivos com melhores técnicas, possibilitando ao paciente uma assistência com o menor risco de complicações (Almeida, et al., 2018).

Entretanto, um estudo realizado entre 2012 e 2016, num hospital público pediátrico localizado na região metropolitana da Zona Leste de São Paulo, registrou 32 notificações de flebite, sendo que 50\% dos pacientes manifestaram flebite grau 3 relacionadas ao PICC. Esses resultados sugerem que em via central pode haver intercorrências como: infecção, obstrução e oclusão, exigindo-se, portanto, a instituição de protocolo para esse cuidado específico. Vale ressaltar que na iminência do primeiro sinal de flebite, deve-se proceder a remoção do cateter e, aplicar compressas frias, seguidas de compressas mornas no local afetado, com elevação do membro de 24 a 48horas, associado a administração de analgésicos e anti-inflamatórios, conforme prescrição médica (Silva, et al., 2020).

Costa, et al., (2016) evidencia que as menores médias de peso e idade gestacional corrigida atrelado ao tempo de permanência do PICC foram associados à infecção da corrente sanguínea (ICS). No que tange à atuação da enfermagem, os autores enfatizam que a inserção do cateter nos RN deve ser uma decisão conjunta entre neonatologista e enfermeiro. Nesse sentido, alguns aspectos devem ser considerados, tais como: o tipo de terapia intravenosa prescrita, a rede venosa, hemograma, nível sérico de plaquetas e condições clínicas do RN.

Além disso, notou-se que o curativo do cateter é trocado nos casos de descolamento e sangramento excessivo ou sujidade na parte interna. A utilização de luva de procedimento e a desinfecção das conexões com solução de clorexidina alcoólica ou swab de álcool 70\%, bem como, a permeabilização do cateter com soluçãofisiológica $0,9 \%$ pré e pós-infusão, foram medidas importantes desenvolvidas pela equipe de enfermagem (Costa, et al., 2016).

Estudo realizado na UTI de um hospital público de Sergipe revelou que em relação à troca de curativo foram avaliadas 2.937 ações, sendo todas executadas por enfermeiros. Esta pesquisa demonstrou que, diferentemente das trocas de equipo, as práticas inerentes à periodicidade da troca de curativo $(93,1 \%)$ e o registro de troca de curativo $(91,7 \%)$ demonstraram altos índices de conformidade. Em contrapartida, a adesão às boas práticas para desinfecção dos conectores, injetores, ampolas e frasco ampolas não exibiram a conformidade geral esperada, de acordo com o bundle (Llapa, et al., 2020). Corroborando esse estudo, pesquisa australiana objetivando instituir uma ferramenta para monitorar o cumprimento da desinfecção dos injetores evidenciou fragilidades dos profissionais de enfermagem, na execução desta prática, atingindo uma conformidade de $60 \%$ (Desra, et al., 2016).

No que se refere à assistência de enfermagem na inserção do PICC, estudo de Rangel, et al., (2019) evidencia que a hidratação venosa, a nutrição parenteral, a antibioticoterapia, a infusão de glicose superior a 12,5\% e administração de drogas 
vasoativas são procedimentos corriqueiros e estão associadas ambiente da UTIN.Nesta perspectiva, a enfermagem tem um função primordial em relação à adoção de medidas farmacológicas ou não, que diminuam a dor associada ao cateterismo. Neste estudo, evidenciou- se que o tempo de permanência foi influenciado pela localização daponta não central, complicações e remoção acidental.

Os bundles representam ferramentas adicionais ao checklist responsáveis por direcionar e subsidiar a equipe de enfermagem a utilizarem medidas de prevenção das infecções relacionadas à assistência à saúde (IRAS), que constitui um conjunto de medidas estratégicas, visando primar pela segurança ao paciente em uso da terapia infusional com o cateter central (Araújo, et al., 2017 \& Manzo et al., 2019).

Neste ínterim, o enfermeiro pode ser considerado facilitador no processo de identificação de riscos de EA, constituindo peça chave nesse processo, dado o seu protagonismo na assistência. Nota-se que à medida que os enfermeiros assumem seu papel de liderança junto à equipe, há evidências de melhoria da assistência, portanto, devem notificar, comparar e mensurar os incidentes e suas consequências. Além disso, o enfermeiro é o responsável técnico pela equipe de enfermagem frente ao seu conselho de classe, e irá responder a possíveis processos judiciais relacionados à ocorrência de EA, respondendo pela omissão, negligência, imperícia e imprudência de qualquer membro da sua equipe (Sorgi, et al., 2019).

\section{Conclusão}

Conclui-se que o enfermeiro exerce um papel essencial na prevenção e controle de infecções, visto que assistem diretamente os pacientes, manipulam e controlam dispositivos, conexões e medicações. O estudo evidenciou a importância de gerenciar riscos inerentes à cateterização, tais como a infecção da corrente sanguínea, em que o enfermeiro deve estar atento desde o momento da inserção até a remoção docateter.

As estratégias recomendadas para redução das infecções relacionadas ao sítio de inserção, está a utilização de bundles, que correspondem a um pacote de medidas que, ao serem praticadas em conjunto, são eficazes na redução das taxas de infecção. Essas medidas já são preconizadas pelo CDC, que destaca a importância do uso de barreira máxima de precaução associada as demais recomendações, para reduzir as chances de colonização do dispositivo no momento da inserção.

Concluímos que o presente trabalho sirva como modelo para uma análise reflexiva dos gestores, profissionais da área de saúde que atuam continuadamente na assistência aos pacientes que utilizam ceteter venoso central, utilizando como reflexões para novos estudos que visem promover um sistema de gestão de qualidade em relação aos cuidados relacionados para este tipo de intervenção.

\section{Referências}

Almeida, T. M., et al. (2018). Prevenção de infecções relacionadas ao cateter venoso central não implantado de curta permanência. Revista enfermagem UERJ. 26(1).

Araújo, F. L., et al. (2017). Adesão ao bundle de inserção de cateter venosocentral em unidades neonatais e pediátricas. Revista da Escola de Enfermagem da USP. 51(2)

Barbosa, J. A. S., et al. (2020). Cateter venoso central de inserção periféricae trombose: experiência em hospital de alta complexidade. Cogitare Enfermagem. $25(8)$.

Brasil. Agência Nacional de Vigilância Sanitária (Anvisa). (2016). Implantação do Núcleo deSegurança do Paciente em Serviços de Saúde. Série Segurança do Paciente e Qualidade em Serviços de Saúde.

Brasil. Agência Nacional de Vigilância Sanitária (Anvisa). (2014). Boletim de Segurança doPaciente e qualidade em Serviços de Saúde nº 12 . Rede Nacional de Monitoração deResistência Microbiana. Relatório da Resistência de Infecções Primárias de Corrente Sanguínea Relacionadas a Cateter em Unidades de Terapia Intensiva.

Brasil. Agência Nacional de Vigilância Sanitária (Anvisa). (2013). Resolução da Diretoria Colegiada-RDC $n^{\circ}$. 36 de 25 de julho de 2013 que institui ações para a segurança do pacienteem serviços de saúde e dá outras providências. Diário Oficial da União.

Brasil. Agência Nacional de Vigilância Sanitária (Anvisa). (2011). Resolução da Diretoria Colegiada $-R D C n^{\circ}$. 63 de 25 de novembro de 2011 . Dispõe sobre os requisitos de Boas Práticas de funcionamento para os serviços de saúde. Diário Oficial da União. 
CDC - Center for Disease Control and Prevention. (2016). Bloodstream infection event (central line-associated bloodstream infection and non-central lineassociated bloodstream infection). Atlanta, GA: Centers for Disease Control and Prevention, 4(2), 1-32.

Costa, C. A. B., et al. (2020). Bundle de Cateter Venoso Central: conhecimento e comportamento de profissionais em Unidades de Terapia Intensivaadulto. Revista Escola de Enfermagem USP. e03629-e03629.

Costa, P., et al. (2016). Fatores de risco para infecção de corrente sanguínea associadaao cateter central de inserção periférica em neonatos. Acta Paulista de Enfermagem. 29(2), 161-168.

Danski, M. T. R., et al. (2017). Complicações infecciosas associadas aocateter venoso central totalmente implantável. Rev. enferm. UFPE on line, 5049-5058.

Desra, A. P., et al. (2016). Aseptic technique for accessing central venous catheters: applying a standardised tool to audit 'scrub the hub'practices. The journal of vascularaccess. 17(3), 269-272.

Llapa, R., et al. (2020). Segurança na troca de equipos e curativospara cateter vascular central: um estudo observacional. Texto \& Contexto- Enfermagem, 29(4).

Manzo, B. F., et al. (2019). Conhecimento e comportamento de profissionais sobreo bundle de cateter venoso central. Revista Brasileira de Enfermagem. 72(1), $50-56$.

Marconi, M. A. \& Lakatos, E. M. (1992). Metodologia do trabalho científico. São Paulo: Editora Atlas, 4(2), 43-44.

Marques, J., Santos, F., Aquino, R. L., Júnior, P. \& Ferreira, N. (2019). Infecção da corrente sanguínea relacionada ao cateter venosocentral. Rev. enferm. UFPE on line. 1-11.

Oliveira, C. G. \& Rodas, A. C. D. (2017). Tecnovigilância no Brasil: panorama das notificações de eventos adversos e queixas técnicas de cateteresvasculares. Ciência \& Saúde Coletiva. 22, 3247-3257.

Ortega, D. B., et al. (2017). Análise de eventos adversos em pacientes internados em unidade de terapia intensiva. Acta Paulista de Enfermagem. 30(2), 168173.

Ramírez, M. M., et al. (2017). Cultura de segurança e eventos adversos em umaclínica de primeiro nível. Enfermería universitaria. $14(2), 111-117$.

Rangel, R. J. M., et al. (2019). Práticas de inserção, manutenção e remoção doCateter Central de Inserção Periférica em neonatos. Revista Pesquisa Cuidado Fundamental (Online), 278-284.

Ribeiro, A. M. N., et al. (2020). Prevenção de infecção relacionada à catetervenoso central: cuidados e conhecimento da equipe de enfermagem. Research, Society and Development. 9(11), e93091110711- e93091110711.

Roque, K. E., Tonini, T., \& Melo, E. C. P. (2016). Eventos adversos na unidade de terapia intensiva: Impacto na mortalidade e no tempode internação em um estudo prospectivo. Cadernos de Saúde Pública. 32, e00081815.

Rynga, D., et al. (2017). Hand hygiene compliance and associated factors among healthcare workers in a tertiary care hospital: Self reported and direct observation. International Journal of Infection Control. 13(1).

Silva, A. P. A., et al. (2018). Atuação do enfermeiro frente à infecção da corrente sanguínea associada ao uso do cateter venoso central. Rev Elet Atualiza Saude. $8(8), 29-36$.

Silva, W. C. R., et al. (2020). Indicador de flebite e cuidados de enfermagemem crianças e adolescentes com cateter central de inserção periférica. Global Academic Nursing Journal, 1(3), e44-e44.

Sorgi, G. M. F., et al. (2019). Implantação de pacote de medidas para prevenção de infecções associadas ao cateter venoso central em crianças: percepção da equipe de enfermagem. Revista Eletrônica Acervo Saúde. 11(4), e238-e238.

Sousa, F. C., et al. (2018). Avaliação dos cuidados de enfermagem com o catetervenoso central em uma unidade de terapia intensiva adulto e pediátrica. Revista de Administração em Saúde. 18(70).

Weeks, K. R., et al. (2011). Prevention of central line-associated bloodstream infections: a journey toward eliminating preventable harm. Current infectious disease reports. 13(4), 343-349.

World Health Organization (WHO). The conceptual framework for theinternational classification for patient safety (ICPS). Geneva: WHO. 